\title{
Desarrollo de valor aǵregado en la auditoría de certificación en organizaciones de alta complejidad en Colombia con sistemas de gestión ${ }^{*}$
}

Development of added value in the certification audit in highly complex organizations in Colombia with management systems

Desenvolvimento de valor acrescentado na auditoría de certificação em organizações de grande complexidade na Colômbia com sistemas de gestão

Iveth Cristina Rodrígue: Cermeño

Universidad Santo Tomás

Cómo citar este artículo: Rodríguez-Cermeño, I. C. (2020). Desarrollo de valor agregado en la auditoría de certificación en organizaciones de alta complejidad en Colombia con sistemas de gestión. Signos, Investigación en Sistemas de Gestión, 12(2), 31-45. Dor: https://doi.org/10.15332/24631140.5935

\footnotetext{
Artículo de resultado de la investigación "Modelo para auditorías de certificación en organizaciones de alta complejidad en Colombia con sistemas de gestión".

** Profesional en Administración y Finanzas. Candidato a Magíster en Calidad y Gestión Integral. Línea de investigación: Gestión de calidad. Universidad Santo Tomás. Correo electrónico: ivethrodriguez@usantotomas.edu.co ivethc_rodriguezc@yahoo.com. oRCiD: https://orcid.org/0000-0002-3083-6330
} 


\section{RESUMEN}

La complejidad es un concepto que aparece en diferentes contextos; no empero, la complejidad se fundamenta al reconocer sus elementos y las relaciones entre ellos. Entender la complejidad en el contexto organizacional es un objetivo preciso de abordar cuando se habla de certificación. Profundizar en las variables existentes dentro de las dinámicas de la organización permitirá la comprensión del funcionamiento de sus sistemas de gestión para la generación de valor desde las auditorías. Este artículo centra su atención en destacar la propuesta investigativa de un modelo para auditorías de certificación en organizaciones de alta complejidad en Colombia con sistemas de gestión. Además, describe cómo a través de sus componentes se propende por la generación de valor como resultado de considerar las características particulares de la organización, los niveles de evolución y progreso de los sistemas, y la interacción de los varios elementos organizativos, todos explorados en la investigación.

Palabras clave: auditoría, auditoría de certificación, complejidad, niveles de madurez, organizaciones de alta complejidad, valor agregado.

\section{ABSTRACT}

Complexity is a concept found in different contexts; nevertheless, complexity is based on the recognition of its elements and the relationships between them. Understanding complexity in the organizational context is a precise objective to address when it comes to certification. Deepening in the existing variables within the organization's dynamics will allow understanding the operation of its management systems for the generation of value from audits. This article highlights the research proposal of a model for certification audits in highly complex organizations in Colombia with management systems and describes how through its components it tends to generate value as a result of considering the particular characteristics of the organization, the levels of evolution and systems progress as well as the interaction of the various organizational elements, all explored in the research.

Keywords: Audit, Certification audit, Complexity, High complexity organizations, Maturity levels, Added value.

\section{RESUMO}

A complexidade é um conceito que surge em enquadramentos diferentes, mas a complexidade baseia-se no reconhecimento dos seus elementos e das relações entre eles. Compreender a complexidade no âmbito organizacional é um objetivo preciso na hora de falar em certificação. Aprofundar nas variáveis verificadas dentro da dinâmica da organização permitirá a compreensão do funcionamento dos seus sistemas de gestão para a geração de valor a partir das auditorías. Este artigo foca a sua atenção em destacar a partir da proposta de pesquisa de um modelo para auditorías de certificação em organizações de grande complexidade na Colômbia com sistemas de gestão e descreve como através dos seus componentes é susceptível de gerar valor como resultado de considerar as características próprias da organização, os níveis de evolução e progresso dos sistemas e a interação dos vários elementos organizacionais, todos eles analisados na pesquisa.

Palavras-chave: Auditoría, Auditoría de certificação, Complexidade, Organizações de grande complexidade, Níveis de maturidade, valor acrescentado. 


\section{INTRODUCCIÓN}

El mundo globalizado y los avances en las tecnologías indiscutiblemente han producido cambios en los países, los gobiernos, la economía, la política, la cultura, los mercados y las preferencias, gustos y necesidades de los usuarios y consumidores. El comercio internacional afecta positivamente la productividad, porque facilita el acceso a capital productivo, insumos de producción, tecnología, estándares de producción y economías de escala en el proceso de expansión hacia nuevos mercados (Fieler, Eslava, \& Yi, 2018).

Asimismo, la globalización reasigna factores de producción hacia sectores que los emplean con mayor eficiencia y tienen ventajas comparativas (Eslava, Haltiwanger, Kugler, \& Kugler, 2012). Además, beneficia a los consumidores, al permitirles acceder a una mayor variedad y calidad de productos a precios más bajos, tras inducir mayores niveles de competencia y escala en el mercado (Hummels \& Klenow, 2005; Departamento Nacional de Planeación [DNP], 2019).

Estos retos de la economía global y los cambios actuales vienen significando que tanto los países como las empresas tengan que trabajar por aumentar su capacidad operativa, tecnológica, financiera y de gestión, de tal suerte que el concepto de la calidad se ve más arraigado gracias a estas exigencias. Como lo afirmó el entonces director de comercio internacional e integración de la Comisión Económica para América Latina y Caribe (CEPAL) Osvaldo Rosales, hay temas nuevos de la agenda global tales como la seguridad en el comercio, los vínculos entre comercio, cambio climático y medio ambiente, e incluso algunos aspectos inherentes a la competitividad, como la certificación de calidad o las buenas prácticas productivas (Rosales, 2009).

Los gobiernos han tenido que incluir en su agenda los temas asociados a la infraestructura de la calidad con la intención de promover la seguridad, la calidad, la confianza, la productividad y la competitividad de los sectores productivos e importadores de bienes y servicios, y proteger los intereses de los consumidores en los asuntos relativos a procesos, productos y personas (Ministerio de Comercio, Industria y Turismo [MICIT], 2008).

En Colombia, con el propósito de impulsar la calidad en los procesos productivos y la competitividad de los bienes y servicios en los mercados, se expidió el Decreto 2269 de 1993, posteriormente modificado por el Decreto 3257 de 2008, que desarrollan el Sistema Nacional de Normalización, Certificación y Metrología del Subsistema Nacional de la Calidad, en el marco de la competitividad y la innovación.

De esta normativa se deriva la política nacional de la calidad tendiente al reconocimiento internacional, a través de la reorganización de la institucionalidad existente en esta materia y del fortalecimiento de las actividades de normalización, acreditación, evaluación de la conformidad, expedición de reglamentos técnicos y metrología. Así, el Subsistema Nacional de la Calidad constituye actualmente un componente fundamental para el efectivo aprovechamiento de los tratados de comercio vigentes, toda vez que permite la inserción de productos colombianos al mercado global, a través del establecimiento de normas y reglamentos técnicos adaptados conforme a las tendencias internacionales (Consejo Nacional de Política Económica y Social [CONPES], 2006). En este contexto, los procesos de certificación, reconocidos por la industria y los sectores económicos, permiten la "demostración de que se cumplen los requisitos especificados relativos a un producto, proceso, sistema, persona u organismo" (Icontec, 2005). A 31 de diciembre del 2017, cerca de 1.058.000 empresas en el mundo se encontraban con certificaciones en la Iso 9001 y 362.510 empresas en la ISO 14001; en América Latina, había un total de 45.541 empresas certificadas en Iso 9001, y 10.301 en IsO 14001; en Colombia, 11.471 empresas estaban certificadas en ISo 9001 y 2.954, en ISO 14001 (ISO Survey, 2018). 
Estas cifras demuestran el potencial crecimiento en la implementación y en la certificación de sistemas de gestión motivados por las exigencias de la globalización. Los clientes y partes interesadas demandan cada vez más organizaciones que sean eficientes y confiables en sus servicios o productos, respondiendo con mayor calidad a la satisfacción de sus necesidades y expectativas. La decisión en las organizaciones de implementar y certificar sistemas de gestión viene siendo reconocida como una buena práctica y una ventaja competitiva.

Obtener una certificación de calidad genera confianza entre las partes por la dinámica constante de revisión y verificación para la determinación de la conformidad de los productos y servicios. Es claro que las organizaciones tienen características y particularidades propias y que están en constante cambio por la tecnificación de procesos, la multiplicidad y diversificación de los negocios, la expansión de los mercados, las transformaciones culturales y la innovación, que al relacionarse todas crean dinámicas de complejidad que deben ser entendidas y consideradas en los procesos de certificación y, especialmente, en las auditorías.

Entender este contexto de complejidad en las organizaciones es un reto para los organismos de evaluación de la conformidad. La clasificación de las empresas en Colombia generalmente se hace por número de empleados, ingresos y sectores según las actividades económicas, eso define más o menos elementos organizacionales para su funcionamiento y operación. Sin embargo, la evolución de las empresas ha llevado también a incorporar el concepto de complejidad en las organizaciones según su estructura, definición y explicación (Flores \& Vanoni, 2016). La problemática está centrada en cómo se entiende la complejidad de las organizaciones en los procesos de certificación. Aunque este ha sido un fenómeno experimentado en las auditorías, no existen definiciones precisas sobre ello y sobre cómo comprenderlo para generar valor.
Este artículo presenta el desarrollo y conclusiones del proceso investigativo realizado para el diseño de un modelo para auditorías de certificación en organizaciones de alta complejidad en Colombia con sistemas de gestión y su aporte en la resolución de la pregunta de investigación: ¿Cómo puede el auditor de certificación de sistemas de gestión evaluar y verificar una organización caracterizada como de alta complejidad? El proceso investigativo con enfoque cualitativo busco la exploración teórica del término de complejidad en el contexto organizativo y su interpretación y análisis para la definición de las características de una organización de alta complejidad.

Producto de ello, nacieron nuevos interrogantes asociados a cómo llevar esos elementos a la comprensión de los auditores de certificación y cómo estos deben emplearse para agregar valor en las organizaciones de alta complejidad. En ese sentido, se realizó la revisión y el análisis de información acerca de evaluación de madurez, incorporando estos conceptos al modelo propuesto. Un reto importante surgió de esta investigación asociado a desarrollar durante todas las fases del modelo el contexto de complejidad en las organizaciones y en cómo desde cada una de ellas el concepto de valor agregado debía ser visible. Para presentar los resultados de la investigación, es preciso abordar en principio la exploración realizada sobre complejidad, por lo que se relaciona a continuación.

\section{Concepto de organizaciones de alta complejidad}

Varios autores han escrito acerca de complejidad; por lo tanto, la existencia del concepto tiene fundamento teórico. Se reconoce la complejidad como un sistema compuesto por un gran número de elementos que interactúan de manera no sencilla, donde todo es la suma de las partes (Wimsatt, 1972). La complejidad se fundamenta al reconocer sus elementos y las relaciones 
entre ellos, donde el entendimiento de estos no lleva al conocimiento absoluto (Goldberg \& Holland, 1988).

Un sistema puede ser complejo según su estructura, definición y explicación (Grunberg, 1978). Estas definiciones fueron revisadas por Flores y Varoni (2016) para determinar su propia definición de complejidad, de donde surge la propuesta de que la organización como sistema complejo es el resultado de las interacciones y retroacciones de los componentes y de estos con el medio cambiante y dinámico. De esta manera, se logra identificar el concepto de variedad de elementos y sus interrelaciones, intencionadas o no.

Un análisis frente a la complejidad externa e interna en el contexto organizacional sostiene que la primera describe la perspectiva del mercado, que se caracteriza por los llamados factores de cambio y flexibilidad (por ejemplo, crecimiento demográfico y cambio demográfico, aumento del consumo de recursos o digitalización), mientras que la segunda describe la perspectiva de la empresa, que se caracteriza por los campos de complejidad y la ocurrencia de dimensiones de complejidad. Los campos de complejidad permiten analizar y estructurar la complejidad interna existente en una empresa. Puede ser descrita por los tres campos principales de complejidad, producto (incluidos los servicios), procesos y organización. Por otro lado, las dimensiones de complejidad son la variedad, heterogeneidad, dinámica y non-transparency (Kluth, Jäger, Schatz, \& Bauernhans, 2014).

Por su parte, Jaques (2014) refiere que la complejidad puede definirse por la cantidad de variables que deben manejarse ante una situación en un momento dado, la claridad y precisión con las que puede identificarse a dichas variables y su ritmo de cambio. El contexto en que el autor presenta la complejidad estáasociado al análisis sobre la teoría, conceptos y principios de la jerarquía de responsabilidad gerencial (JRG), y a cómo aplicar el liderazgo gerencial para responder a los retos del siglo actual; por esa razón, la definición de las JRG parte de la comprensión de la estructura jerárquica gerencialsubordinados, y las relaciones de trabajo y funciones.

Estos aportes teóricos abordados en profundidad, junto con la exploración de otros elementos organizativos, permitieron la definición propia de organizaciones de alta complejidad, tal como se presenta a continuación: una organización de alta complejidad, por su estructura y misionalidad, tiene una dinámica especializada para interrelacionar entre síla variedad y cantidad de elementos organizativos con los cuales funciona y que representan una orientación para el alcance del sistema de gestión. La organización de alta complejidad está caracterizada por tener una función central y varias funciones secundarias y auxiliares; puede tener una o más líneas de negocios en un mismo sector productivo o en varios; el número de sitios que la componen y su expansión geográfica es representativo; puede tener diversos grupos de interés y un sinnúmero de procesos o actividades que apalancan sus funciones productivas o de servicios y las secundarias y auxiliares; y responde a reglamentaciones variadas, entre otras características.

\section{METODOLOGÍA}

El proceso investigativo representado gráficamente en la figura 1 tuvo un enfoque cualitativo, teniendo como eje central la experiencia propia del investigador en auditorías de certificación y la definición empíricamente formada acerca del significado de una organización de alta complejidad.

Teniendo en cuenta que se desconocía si en efecto el concepto de alta complejidad en el contexto organizativo ha sido explorado, fue necesario realizar una búsqueda teórica acerca del término complejidady de la relación de elementos que pudiesen determinar las características de las organizaciones de alta complejidad. 
Figura 1. Diagrama de la ruta de investigación

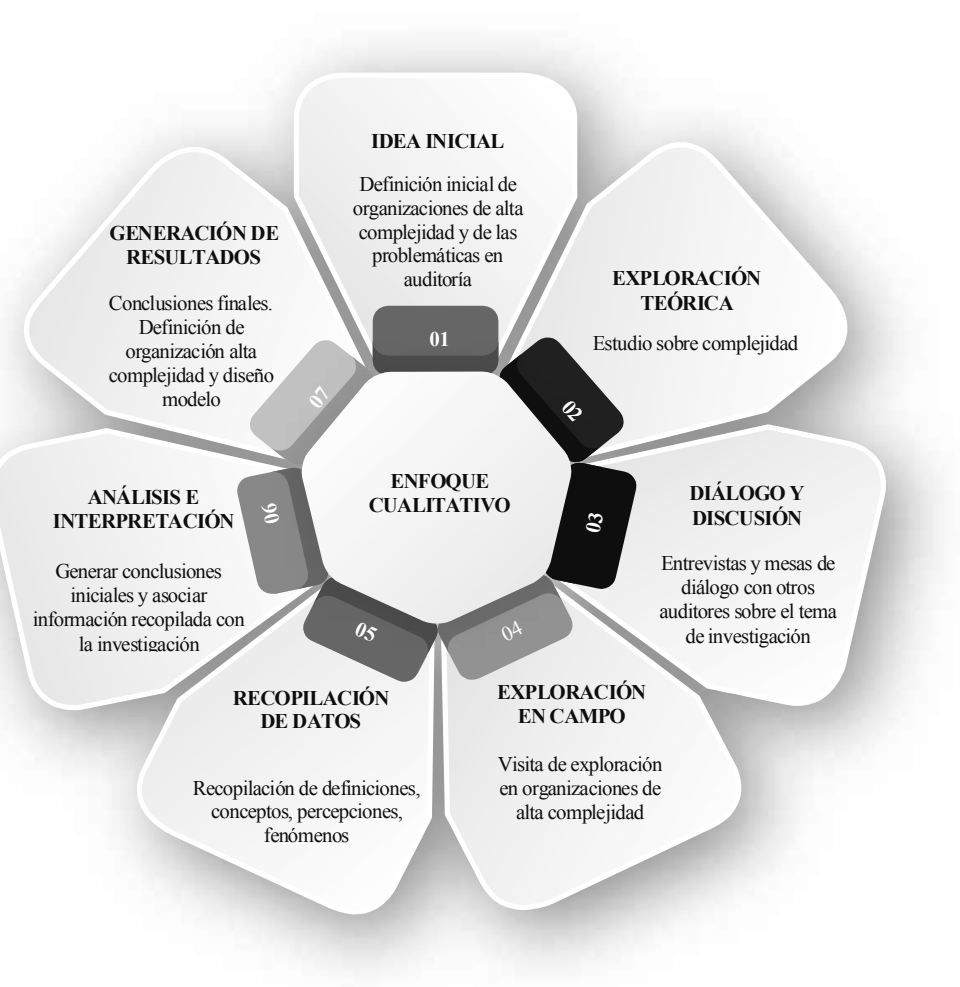

Fuente: elaboración propia.

De dicho proceso, resultaron las primeras versiones del modelo, pero era imperativo validar contextualmente la propuesta. La revisión de la perspectiva del fenómeno de complejidad en el contexto de las auditorías de certificación, así como la discusión acerca de las problemáticas encontradas en las etapas de planificación y ejecución de las auditorías, fue abordada entonces de dos formas: 1) una consulta a siete expertos en materia de auditoría en diferentes momentos de la investigación y 2) una visita a una organización de alta complejidad.

En la primera, tres expertos participaron en una fase inicial a través de una encuesta vía correo electrónico —dos de forma presencial en mesa de trabajo y los otros dos, a través de diálogos generados informalmentey, luego, mediante el envío por correo electrónico del modelo y sus herramientas diseñadas para su lectura y comentarios de acuerdo con unas preguntas preparadas con anterioridad. Por su parte, la visita se llevó a cabo en una caja de compensación ubicada en Bogotá, con más de 17 años de certificación en su sistema de gestión de calidad y alcance a varias de sus líneas de negocio (salud, educación y capacitación, recreación, servicios financieros y de turismo, incluido alimentos y bebidas), con cerca de 42 sitios permanentes donde presta sus servicios. Para auditarla, se definieron alrededor de 45 días con un equipo auditor (compuesto aproximadamente por 12 auditores).

Con esta organización se reflexionó acerca de la vivencia en sus auditorías de certificación y se retroalimentó frente a su percepción del modelo propuesto, que al momento de la visita tenía una versión ajustada con los aportes de algunos de los expertos. 
Las definiciones de organizaciones de alta complejidad, el diseño de las etapas del modelo y la propuesta de escalas de caracterización y madurez fueron resultado del análisis y la interpretación de la información recopilada durante el proceso investigativo.

\section{RESULTADOS}

En la figura 2 se muestra la representación gráfica del modelo para auditorías de certificación en organizaciones de alta complejidad en Colombia, compuesto por cinco fases: conocimiento de la organización; preparación y planificación; ejecución; resultados y entregables, y autoevaluación.

El modelo ha sido diseñado manteniendo el enfoque del ciclo PHN, aunque esperando que las fases se interrelacionen entre ellas y posibiliten la generación de valor a partir de la comprensión de las características de la organización de alta complejidad. En la fase 1, conocimiento de la organización, se hace referencia a la exploración y conocimiento profundo de la estructura y dinámica organizacional a través de sus características propias y particulares.

Figura 2. Representación gráfica del modelo final

01 Conocimiento de la Organización

- Reuniones de contextualización o resumen organizacional

- Indagaciones de expectativas de auditoría. Lista de deseos

- Aplicación de escalas y determinación

05 Autoevaluación

- Aciertos y desaciertos del plan de auditoría

- Lecciones aprendidas

- Recomendaciones al próximo equipo auditor

- Retroalimentación del cliente (organización de alta complejidad)

04 Resultados y entregables 04

- Hallazgos

- Informe de auditoría

- Redacción y uso gramatical

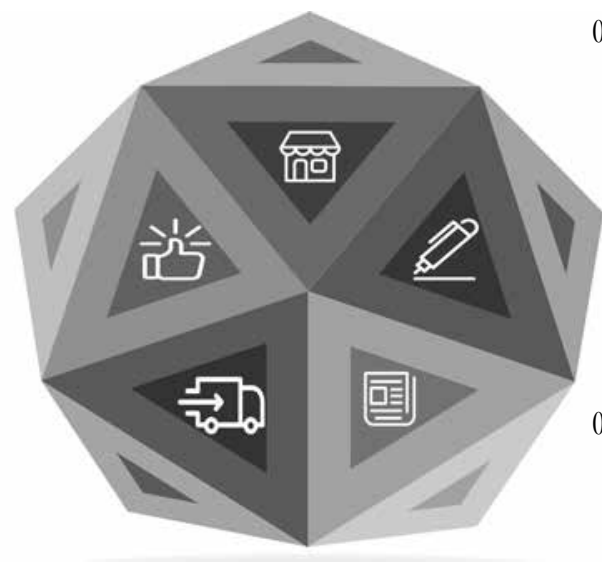

02 Preparación y planificación

- Reuniones equipo auditor con enfoque de red colaborativa

- Estructuración de plan de auditoría

- Definición/redefinición de programa de auditoría

03 Ejecución

- Recopilación y análisis de la información: calidad, cantidad y muestreo

- Balances de auditoría

Fuente: elaboración propia.

En ella se busca de forma preliminar identificar el nivel de madurez del sistema o sistemas de gestión para orientar las actividades de auditoría y la forma de abordar la organización con el sentir de sus expectativas en cuanto a la auditoría y la certificación. Una organización de alta complejidad, como se definió en la introducción, tiene muchas variables que considerar y entender, por lo que es imperativo una mayor profundidad en esta etapa.
No es lo mismo auditar una empresa familiar que produce envases plásticos, por ejemplo, con una sola sede y que vende al por mayor sus productos a nivel regional por Internet, a auditar una empresa que tiene más de 1.000 empleados, varias sedes ubicadas en diferentes sitios a nivel nacional y que, además de la producción, comercializa y ofrece servicios de asesoría para el uso y conservación de envases. Tampoco se puede comparar 
auditar una organización de tipo privado que realiza proyectos de infraestructura local, con auditar una entidad pública regional, que hace la ejecución de proyectos de infraestructura por proyectos de inversión en los diferentes municipios, con un personal contratista de casi el $80 \%$ del total de personas, además de la regulación que debe cumplir y que depende del lugar y jurisdicción de la obra.

¿Cómo abordar entonces la auditoría a estas organizaciones y además generar valor? El primer elemento que plantea esta fase es la aplicación de escalas de caracterización y de madurez como referente para comprender la organización y orientar el ejercicio auditor. Cuando se hizo la revisión teórica de algunos enfoques de medición de madurez se estudió la investigación realizada por Kluth, Jäger, Schatz y Bauernhans (2014), acerca de la evaluación de los sistemas de gestión de complejidad, cuyo aporte indica que la madurez implica un progreso evolutivo en la demostración de habilidades específicas o el logro de objetivos, desde un estado inicial donde se consideran pocas habilidades con respecto a la gestión de la complejidad, hasta un estado final, que es completo, optimizando los recursos de la empresa para lograr el objetivo de armonización de la complejidad interna y externa.

Este es uno de los efectos que se busca con el modelo y las escalas propuestas. Agregar valor desde ellas significa poder hacer un análisis de dónde se encuentra la organización de alta complejidad al iniciar un ciclo de certificación y que, durante la auditoría, el enfoque esté orientado a realimentar frente a las brechas o potencialidades que deban abordarse por la organización, es decir, concluir, de un estado inicial, un estado de evolución.

En este análisis vale la pena referir el modelo de empresa familiarmente responsable (EFR), desarrollado por la Fundación MásFamilia (Madrid, España), que despliega un sistema de puntuación y clasificación de las organizaciones representado en la mejora continua de las variables de conciliación (aspecto base del modelo).
De esta forma, se determinan cuatro grupos de empresas: escépticas, comprometidas, proactivas y excelentes. En el documento normativo 1000-3 Requisitos cuantitativos. Sistema de puntuación y clasificación para grandes y medianas organizaciones, MásFamilia (2014) presenta un análisis del tiempo promedio de evolución que una empresa puede tener en su modelo, señalando que una evolución entre el nivel escéptico y el proactivo se produce tras dos ciclos de certificación, seis años, y de este nivel al máximo la evolución resulta en dos ciclos adicionales. Cabe aclarar que el enfoque del modelo EFR está pensado para implementarse de manera progresiva, por lo que el análisis de tiempo responde a la definición de indicadores definidos para tal efecto. Aunque las proyecciones de tiempo para la evolución y progreso presentado por MásFamilia (2014) no fueron tenidos en cuenta para la definición de las escalas de madurez, su aportación está dada en torno a la demostración de que es posible definir escalas de madurez para evaluar la evolución de un sistema de gestión.

Por ahora, la aplicación de las escalas de madurez está pensada para la orientación de la auditoría y, de forma posterior, para que sean considerados los descriptores al generar los hallazgos de auditoría. En este sentido, también fue contemplado un análisis sobre las orientaciones para llegar al éxito sostenido presentados en la Iso 9004:2018, que en su propósito busca ir más allá del cumplimiento de los requisitos de un sistema de gestión como tal.

Su fundamento se encuentra en que la organización reconozca las condiciones del entorno y las exigencias de cambio en la que se desempeñan para alcanzar el éxito sostenido de manera complementaria a los sistemas de gestión. Por ejemplo, considerar que el contexto de la organización se mueve en un entorno continuamente cambiante lleva a pensar en temas como la globalización, la competencia, la innovación y los avances tecnológicos; o que en las agendas de los directivos deben incluirse los impactos causados a las partes interesadas, entendidas no solo como los clientes sino en la sociedad o la comunidad. 
Esta concepción se fortalece con la revisión realizada a los Estándares GRI publicados por el Global Sustainability Standards Board (GSSB, 2016), que son referentes para la elaboración de informes de sostenibilidad, dado que atañen a los temas de impacto económico, ambiental y social. A modo personal, su mayor aporte es que entiende la organización como un todo; por supuesto, su propósito es publicar los impactos generados en las materias ya mencionadas, abordados desde elementos organizativos que permiten considerar su evolución medida desde la sostenibilidad.

Desde ese punto de vista, fue posible encontrar descripciones que le dieran claridad a los elementos constitutivos de la escala de madurez propuesta desde el modelo. Los Estándares GRI se encuentran divididos en dos grandes grupos: los estándares universales, que incluyen los fundamentos, la información contextual sobre la organización y el enfoque de gestión, y los estándares específicos, que desarrollan lo específico de los aspectos económicos, ambientales y sociales.

De la revisión de los estándares en las escalas fueron utilizadas las consideraciones en relación con la identificación de servicios, negocios o mercados atendidos; las definiciones de contexto de organización y enfoque de gestión, grupos de interés; la identificación y gestión de necesidades y expectativas; la gestión sobre los riesgos y oportunidades, y el enfoque de evaluación de la gestión y mejora.

\section{Fase de preparación y planificación}

Luego de hacer un entendimiento de la organización y su contexto e identificar de forma preliminar el nivel de madurez de la organización de alta complejidad, se propone desde el modelo la potencialización de las herramientas de planificación con la utilización de los resultados de aplicación de las escalas. Para efectos de este artículo se concentrará la atención en el programa de auditoría de forma particular. La GTC-Iso 19011:2018
(Directrices para la auditoría de los sistemas de gestión) refiere que el programa de auditoría establece los acuerdos para un conjunto de una o más auditorías planificadas para un periodo determinado y dirigidas hacia un propósito específico.

El alcance de un programa de auditoría debería basarse en el tamaño y la naturaleza del auditado, asícomo en la naturaleza, funcionalidad, complejidad, tipo de riesgos y oportunidades, y nivel de madurez de los sistemas de gestión que se van a auditar (Iso 19011:2018, Req. 5.1, p. 7). La definición menciona dos elementos que se han abordado en la investigación, complejidad y madurez. En ese sentido, se plantea que el programa de auditoría también considere las escalas de caracterización y madurez para su definición, dado que, conforme el estado de la organización de alta complejidad frente a sus sistemas de gestión, el programa de auditoría orientará la dinámica general para el ciclo de auditoría.

A continuación, se hará referencia a ello. Un programa de auditoría para una organización de alta complejidad caracterizada en un proceso de certificación inicial, con una escala de madurez en nivel básico, debería propender por desarrollar una auditoría con enfoque de tipo orientador, donde los requisitos que se auditarán sean aquellos mínimos para demostrar la estructura base del sistema o sistemas de gestión establecida por la organización.

Los niveles de profundización de la auditoría serán basados en el entendimiento de una organización que apenas se encuentra comprendiendo los requisitos del sistema o los sistemas de gestión. Es posible que los sistemas de gestión se modulen de manera independiente, por lo que el programa de auditoría tendrá que generarse para cada uno y aun no sea posible generar uno integrado. A este tipo de programa de auditoría se llamará beginner y normalmente corresponderá al ciclo de certificación inicial.

Por otro lado, un programa de auditoría para una organización de alta complejidad, con una escala de 
desempeño y madurez intermedio, podría pensarse que se encuentra en su segundo ciclo de certificación con un mayor entendimiento de los requisitos del sistema o sistemas de gestión. En este caso, el programa de auditoría podrá relacionar los requisitos básicos de cada sistema de gestión para auditarlos de manera transversal e incorporar de manera específica los que sean particulares de cada sistema.

Es posible generar una dinámica donde la comprensión de la organización permita una auditoría con enfoque hacia la integración o combinación de los requisitos, sin considerar un sistema integrado en su totalidad. Este programa de auditoría hará referencia al tipo junior.

En un escenario donde la organización de alta complejidad alcance altos niveles de evolución y excelencia en su sistema o sistemas de gestión, podrá demostrar una mayor sinergia para el cumplimiento de los requisitos, de manera que la auditoría fácilmente podrá evaluar el sistema como uno solo y con la alineación eficaz con la estrategia de la organización. Los requisitos de cada sistema de gestión operan entre ellos, permitiendo darle el nivel de profundización a lo particular, lo que conlleva a que la determinación de los momentos para auditarlos dentro del ciclo de certificación no obligue a tener que verificarlos en todas las etapas de auditoría.

Este programa de auditoría corresponderá al tipo advanced. También se contemplaron dentro de la tipología de programas de auditoría dos clasificaciones adicionales que están orientadas a organizaciones que superan los niveles de madurez de sus sistemas de gestión y que, por ende, han empezado a incorporar otros referentes como modelos de excelencia, éxito sostenido y continuidad de negocio. Para ellos se ha establecido los tipos higher y upper. En la figura 3, se presenta la propuesta sobre el programa.

Figura 3. Representación del programa de auditoría

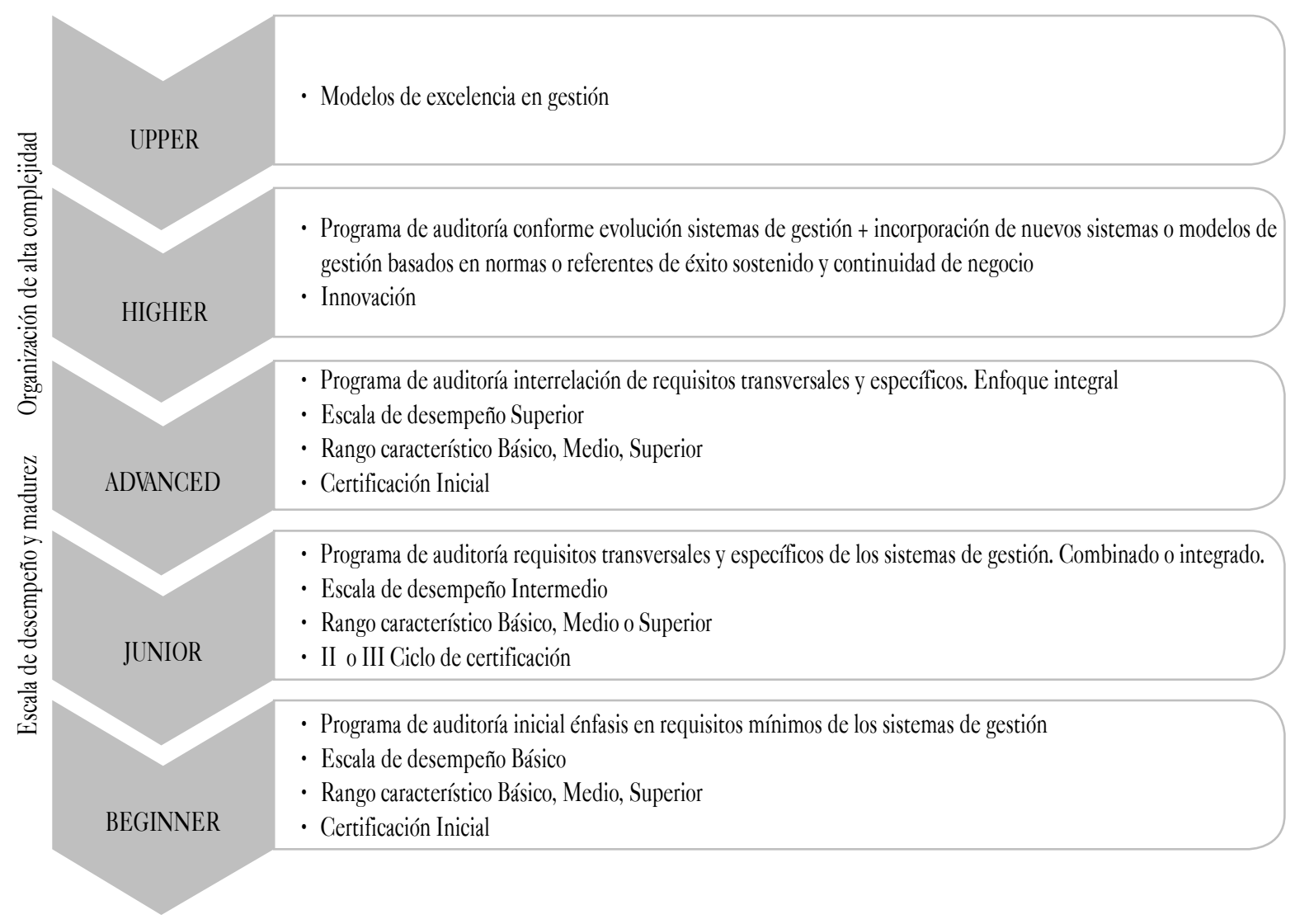




\section{Fase de ejecución}

En esta fase, la generación de valor se ve representada principalmente en cómo los auditores determinan eficazmente los recursos adecuados y suficientes para lograr los objetivos del ejercicio, basándose en una evaluación de la naturaleza y complejidad de las actividades, las restricciones de tiempo y los recursos disponibles. La identificación, análisis, evaluación y síntesis de la información son aspectos relevantes para asegurar que las conclusiones y resultados son objetivos y se dan bajo principios de imparcialidad y coherencia.

No obstante, el nivel de complejidad de la organización puede determinar también el volumen de información que pueda existir en la organización, por lo que el auditor debe dedicar un tiempo a reconocer qué tanta información tiene y cuál es su nivel de documentación. La calidad de la información estará determinada por su fiabilidad, completitud y coherencia, por lo que el modelo hace un examen al tema de la recolección y análisis de información a partir del entendimiento organizacional.

Uno de los riesgos de auditoría que identifica la GTC-ISO 19011 está asociado a la objetividad de los resultados, para lo cual es necesario asegurar las técnicas apropiadas para el muestreo de la información. Considérese una organización con 1.800 empleados distribuidos en 45 sitios permanentes, en la que el $60 \%$ del personal realiza tareas operativas de las unidades de negocio; asumiendo un error de muestra y una proporción de éxito aproximado de 5\%, se establece una muestra de 94 personas que se debe verificar.

Si lo que el auditor va a verificar es participación en jornadas de formación y evaluación del desempeño, esta es una muestra que fácilmente podrá corroborar en listas de asistencia. Pero el nivel de profundidad de la revisión en términos de la eficacia de la formación para el fortalecimiento de las competencias requerirá un tiempo mayor para examinar el número establecido, dado que debe revisar con mayor nivel de detalle el aporte de la formación a los perfiles y los procesos de valoración y fortalecimiento de competencias.

Esta ejemplificación es un escenario que se encuentra cotidianamente en una auditoría; de ahí la pertinencia de referirse desde el modelo al empleo de herramientas que aporten en la decisión de la muestra que se va a considerar para recopilar y analizar información relevante para la determinación de conformidad. En ese sentido, el modelo hace referenciación de estándares como la GTCISO-TR 10017 Orientación sobre técnicas estadísticas o la ISO/IEC 17028 (Evaluación de conformidad: directrices y ejemplos de esquemas de certificación para servicios, que se asemeja a sistemas de gestión en lo intangible y presenta orientaciones para realizar muestreo) o como la NTC-ISO 5667-9:2018 (Gestión ambiental).

Un ejemplo de ello es la calidad del agua; para ello, se realiza un muestreo considerando la utilización de herramientas ya existentes como la Caja de herramientas del Departamento Administrativo de la Función Pública (DAFP), específicamente en lo relativo al cálculo de la muestra. Cualquiera de ellas puede ser empleada por el auditor, asícomo la definición de criterios para realizar el muestreo. En una auditoría de certificación en organizaciones de alta complejidad, es coherente que un criterio de auditoría pueda establecer en qué tipo de información documentada se podrá hacer un muestreo aplicando el enfoque estadístico, y en cuál podrá emplearse un muestreo basado en juicios, en este último, con la claridad que se asumirá un mayor riesgo de la incertidumbre en los resultados y conclusiones de la auditoría.

Como se ha venido señalando, una organización de alta complejidad se podrá caracterizar por la "complejidad" de los requisitos en temas asociados a reglamentación, o a la misma definición y diversidad de negocios, o por la interacción de los procesos de la organización, los elementos del sistema o sistemas de gestión y ambiente organizacional y el factor humano. Este conocimiento de las características y factores particulares de una organización de alta 
complejidad será base para definir y poner en práctica un criterio de muestreo basado en juicios.

\section{Fase de resultados y entregables}

Partiendo de las connotaciones de las fases anteriormente explicadas, se resume que la generación de valor se da cuando se logran concentrar todos los análisis realizados durante la auditoría para que sean presentados a la organización de alta complejidad, y estos sean entendidos y verdaderamente empleados para la mejora continua de los sistemas de gestión.

Por este motivo, el modelo concluye que, al reportar los hallazgos de auditoría, estos deben disponerse en perspectiva del análisis que el equipo auditor logró hacer durante la ejecución de la auditoría, teniendo como norte no solo el grado de cumplimiento de los requisitos, sino el nivel de madurez encontrado para que se retroalimente a la organización frente a las brechas, los riesgos y los impactos.

Pero también es pertinente pensar en la generación de hallazgos según la audiencia. En la fase de conocimiento del cliente, se propone la construcción de la "lista de deseos" del cliente, que, aunque no nos referimos a ella en este artículo, sí se trae a colación en el sentido de que, para generar hallazgos según la audiencia, esta lista es pertinente para considerar hallazgos de atención de la alta dirección para la toma de decisiones y hallazgos de interés común.

En los resultados es conveniente incluir también un análisis generalizado del nivel de madurez encontrado. Por consiguiente, es preciso que la auditoría analice las variables que determinan en qué punto está una organización, pero también en qué condiciones está para abordar el futuro (PwC, 2013). Es clave la elaboración de informes que aporten una visión integral de la organización y que aseguren el entendimiento de los hallazgos en el lenguaje de la organización para su comprensión y utilidad.
Aquí se condensa por escrito la generación de valor en la opinión general del juicio profesional de los auditores. El empleo correcto del lenguaje, el estilo de redacción, las reglas gramaticales y lingüísticas serán el camino para la interpretación sin ambigüedades de los resultados de auditoría por parte de las organizaciones de alta complejidad.

Fakhfakh (2015) hace un análisis acerca de una revisión de informes de auditoría financiera desde donde concluye que las características de un informe de auditoría deben cumplir con las características de legibilidad, manejo lingüístico, comprensibilidad, precisión, consistencia, claridad y longitud.

\section{Fase de autoevaluación}

Para terminar el modelo y referir el enfoque de generación de valor, esta fase invita a hacer una reflexión de la organización, así como el enfoque y la logística manejada durante la auditoría. Se lleva a cabo una revisión de la medida en que se lograron las dinámicas esperadas de auditoría y si el enfoque fue apropiado. Es momento de retroalimentar y dejar aprendizajes para la mejora.

El modelo hace varias propuestas: la identificación de aciertos y desaciertos de la auditoría; la revisión de dónde hubo posibles fallas y considerar recomendaciones que coadyuven a seguir profundizando más en las dinámicas de la organización de alta complejidad; indagar con los auditores y con la misma organización de alta complejidad acerca del enfoque, la planificación y la ejecución de la auditoría y si sus expectativas fueron cumplidas; la recopilación y puesta en conocimiento de lecciones aprendidas con un análisis consciente de aquellos elementos o situaciones que tuvieron éxito y cuáles no; finalmente, la generación de propuestas de mejora concentradas en las etapas de la auditoría y cuáles acciones se pueden contemplar para el cierre de brechas y el fortalecimiento del ejercicio. En la figura 4 se contextualizan los cinco caminos propuestos en la autoevaluación. 
Figura 4. Componentes de la autoevaluación

\section{Percepción del equipo auditor frente al ejercicio}

Revisión del plan de auditoría y su ejercicio aciertos y desaciertos

\section{Identificación de análisis de lecciones aprendidas}

\section{Generación de recomendaciones de mejora para próximas auditorías}

\section{Retroalimentación con el cliente (organización)}

Fuente: elaboración propia.

\section{DISCUSIÓN}

En la revisión de expertos frente al modelo se discutió acerca de cómo llevar a la realidad la aplicación de las escalas de caracterización y de madurez de una forma práctica, para que el auditor le diera la utilidad esperada. Ante este hecho, el modelo diseñó dos herramientas en Excel para su aplicación con formulación para facilidad en su diligenciamiento; no obstante, no han sido aplicadas en un ambiente real de auditoría para lograr contextualizar los resultados de su utilización y eficacia, con el fin de darle enfoque a la auditoría y generar valor.

Otra de las discusiones dadas fue en relación con los tiempos para la auditoría, aunque el modelo no considera las fases previas a su realización como la programación de agendas y asignación del equipo auditor; en efecto, esta es una circunstancia que aqueja la auditoría, dado que abordar tantas variables y elementos de complejidad en el ejercicio se hace más difícil, porque los tiempos no son suficientes y el auditor en la realidad carece de formas para solucionarlo. En ese sentido, la distribución de tiempos, la recopilación y análisis de información, y la generación de valor más allá de la simple revisión del cumplimiento de los requisitos del sistema o los sistemas de gestión es una tarea ardua.

\section{CONCLUSIONES}

Generar valor agregado en la auditoría hace referencia al entendimiento de la organización de alta complejidad (a sus particularidades) y a considerar sus expectativas frente al aporte de la auditoría para alcanzar los retos de su organización en el mundo globalizado o para el éxito sostenible. Esto significa hacer un análisis de las capacidades de la organización apalancadas en la verificación de la conformidad de los modelos de gestión para el logro de los objetivos de crecimiento, sostenibilidad y mejora.

Las escalas de caracterización de organizaciones de alta complejidad y de madurez de sistemas de gestión pueden llegar a ser un valioso aporte en la auditoría, teniendo presente que su fundamento es el de entender el nivel de evolución de la organización y la comprensión 
de sus rasgos particulares, base para la generación de conclusiones y aportes para alcanzar el éxito sostenido. No obstante lo anterior, es requerido ponerlas a prueba en un ambiente real de auditoría, con motivo de validar su pertinencia y eficacia e identificar posibles obstáculos para su utilización.

\section{REFERENCIAS}

Consejo Nacional de Política Económica y Social (CONPES). (2006). Documento CONPES 3446: Lineamientos para una politica nacional de la calidad. Bogotá: CONPES.

Departamento Nacional de Planeación (DNP). (2019). Bases del Plan de Desarrollo 2018 - 2022. En un mundo de posibilidades: aprovechamiento de mercados internacionales y atracción de inversiones productivas. Bogotá: DNP. Recuperado de https://www.dnp.gov. co/Plan-Nacional-de-Desarrollo/Paginas/Bases-delPlan-Nacional-de-Desarrollo-2018-2022.aspx

Eslava, M., Haltiwanger, J., Kugler, A., \& Hluger, M. (2013). Trade and market selection: Evidence from manufacturing plants in Colombia. Review of Economics Dynamics, 16(1), 135-158. Dor: https://doi. org/10.1016/j.red.2012.10.009

Fakhfakh, M. (2015). The readability of international illustration of auditor's report: An advanced reflection on the compromise between normative principles and linguistic requirements. Journal of Economics, Finance And Administrative Science, 20(38), 21-29. DOI: 10.1016/j.jefas.2015.02.001

Fieler, A., Eslava, M., \& Yi, D. (2018). Trade, quality upgrading, and input linkages: Theory and evidence from Colombia. American Economic Review, 108(1), 109-146. Dor: https://doi.org/10.1257/aer.20150796

Flores, M., \& Vanoni, G (2016). Competencias directivas requeridas por los CEO ante la complejidad de las organizaciones del siglo XXI. Suma de Negocios, 7, 117. DoI: https://doi.org/10.1016/j.sumneg.2016.02.005

Fundación MásFamilia. (2014). Documento normativo EFR 1000-3 Modelo de gestión EFR empresa. Requisitos cuantitativos y sistema de puntuación para Grandes y Medianas Organizaciones. Madrid: Fundación MásFamilia.

Global Sustainability Standards Board (GSSB). (2016). Estándar GRI 101: Fundamentos. Ámsterdam, Países Bajos. Disponible en www.globalreporting.org/ standards

Goldberg, D., \& Holland, J. (1988). Genetic algorithms and machine learning. Machine Learning, 3(2), 95-99.

Grunberg, E. (1978). Complexity' and 'Open systems' in economic discourse. Journal of Economic Issues (Association For Evolutionary Economics), 12(3), 541-560. Dor: https://doi.org/10.1080/00213624.19 78.11503553

Hummels D., \& Klenow P. J. (2005). The variety and quality of a nation's exports. American Economic Revierw, 95(3),704-723. DoI: https://doi. org/10.1257/0002828054201396

Instituto Colombiano de Normas Técnicas y Certificación (Icontec). (2005). Norma Técnica Colombiana NTC-ISO/IEG 17000 Evaluación de la conformidad. Vocabulario y principios generales. Bogotá: Icontec.

Instituto Colombiano de Normas Técnicas y Certificación (Icontec). (2018). Guía Técnica Colombiana 
GTC-ISO 19011 Directrices para la auditoría de los sistemas de gestión. Bogotá: Icontec.

Instituto Colombiano de Normas Técnicas y Certificación (Icontec). (2018). Guia Técnica Colombiana GTC-ISO 9004. Calidad de una organización. Orientación para lograr el exxito sostenido. Bogotá: Icontec.

Instituto Colombiano de Normas Técnicas y Certificación (Icontec). (2005). Norma Técnica Colombiana NTC-ISO/IEC 17000 Evaluación de la conformidad. Vocabulario y principios generales. Bogotá: Icontec.

International Organization for Standardization (ISO). (2018). Iso Survey 2017. Recuperado de https://www. iso.org/the-iso-survey.html

Jaques, E. (2014). La organización requerida. Un sistema integrado para crear organizciones eficaces y aplicar el lideraxgo gerencial en el siglo Xxı. Buenos Aires: Granica.

Kluth, A., Jäger, J., Schatz, A., \& Bauernhansl, T. (2014). Evaluation of Complexity Management Systems Systematical and Maturity-Based Approach. Procedia cIRP, 17, 224-229. Dor: https://doi.org/10.1016/j. procir.2014.01.083
Fundación Másfamilia (2014). Documento normativo efr 1000-3 Modelo de gestión efr empresa. Requisitos cuantitativos y sistema de puntuación para Grandes y Medianas Organizaciones. Madrid: Fundación Másfamilia.

Ministerio de Comercio, Industria y Turismo (MICIT). (2008). Decreto 3257, por el cual se modifica parcialmente el Decreto 2269 de 1993 y se dictan otras disposiciones. Bogotá. Recuperado de https://diariooficial.vlex.com.co/vid/decreto-353768510.

PwC. (2014). Temas candentes: La auditoría del futuro yel futuro de la auditoría. Recuperado de https://www. pwc.es/es/publicaciones/auditoría/assets/informetemas-candentes-auditoría.pdf.

Rosales, O. (2009). La globalización y los nuevos escenarios del comercio internacional. CEPAL, 97, 78. DoI: https://doi.org/10.18356/e1ceba76-es

Wimsatt, W. (1972). Complexity and Organization. Proceedings of the Biennial Meeting of the Philosophy of Science Association, 67-86. Dor: https://doi. org/10.1086/psaprocbienmeetp.1972.3698961 